\title{
Video Communication for Using of Telemedicine in Traffic Accidents
}

\author{
Sakineh Hamidi \\ School of Allied Medical Sciences, Alborz University of Medical Sciences, Karaj, Iran \\ Email address: \\ sakineh.hamidi@yahoo.com (S. Hamidi) \\ To cite this article: \\ Sakineh Hamidi. Video Communication for Using of Telemedicine in Traffic Accidents. International Journal of Biomedical Science and \\ Engineering. Vol. 6, No. 1, 2018, pp. 7-19. doi: 10.11648/j.ijbse.20180601.12
}

Received: January 17, 2018; Accepted: February 11, 2018; Published: March 28, 2018

\begin{abstract}
Since the emergence of telegraph and telephone technologies in the 19th Century, doctors have been communicating and consulting with each other over long distances. Telemedicine, as distance healing was first highlighted in 1970, when Thomas Bird wrote about patient care in which physicians were able to examine their patients by using telecommunication technologies. In short, telemedicine can simply involve two health professionals discussing a case over the telephone, or be as sophisticated as using the satellite technology to broadcast a consultation between providers at facilities in two countries, using video conferencing equip men. Telemedicine has the potential to reduce differences in the lives of people, especially those living in remote areas, away from hospitals and thus deprived of quality and timely medical care. The main role of telemedicine is to provide rapid access to experienced health care professionals at a distance using telecommunications and information technologies, no matter where the patient is located. The spectrum of technology used in telemedicine is broad, ranging from simple phone, faxes and emails, to satellite-based relay transfers and state-of-the-art computer and videoconferencing facilities. We divide video communication in telemedicine into videoconferencing and telepresence. Videoconferencing (VC) is defined as a real-time, live, interactive program in which one set of participants are at one or more locations and the other set of participants are at another location. VC permits interaction, including audio and/or video, and possibly other modalities, between at least two sites. Using $\mathrm{VC}$, technical requirements regarding quality are not usually very demanding. Telepresence, on the other hand, widens the purpose of practice beyond pure communication and has clear requirements, mainly concerning the quality and control of the picture as well as time latency. Surgery has entered the computer age with the advent of video laparoscopy. Magnified and computer-enhanced video image provided surgeons with better exposure and visualization of the abdomen. However, a decade after the launch of the new technology it is still poorly accepted. Most laparoscopic procedures are difficult to teach and learn, in addition, the learning curve is very flat. Obvious weaknesses of new technology are: unstable camera platforms, limited motion of straight laparoscopic instruments, twodimensional imaging and poor ergonomics for the surgeon. Since the introduction of video laparoscopic cholecystectomy, surgeons have speculated that computers, 3-D imaging, and robotics could overcome these pitfalls of laparoscopy.
\end{abstract}

Keywords: Video-conferencing, Telemedicine, Traffic Accident

\section{Video-Conferencing}

Video-conferencing (VC) is a specialized form of telemedicine that uses technology to provide real-time visual and audio patient assessment. (Kitamura et al., 2010) Originally, VC was developed to connect physicians with patients located in isolated areas at which climatic or geographical conditions render provider or patient transportation difficult and costly (Sezeur, 1998), resulting in inequalities in patient care (Woottoon, 1999). Examples of
VC practice in telemedicine are: interdisciplinary team meetings, teleconsultation, and tele-education.

\subsection{Interdisciplinary Team Communication}

Interdisciplinary teams (IDTs) are an essential aspect of modern organizational work and are an important facilitator in achieving positive, cost-effective outcomes in various 
organizational settings (Procter \& Currie, 2004). Nowhere is interdisciplinary team communication more important than in health care settings, as the complex nature and demands of the health care work environments require the expertise and knowledge of differing individuals or specialists who can work together to solve multifaceted and complex patient care problems (Heinmann \& Zeis, 2002). Research has demonstrated that interdisciplinary teamwork can improve the diagnostic and prognostic abilities of health professionals, more than individual health professionals working alone, and is also essential for the prevention of medical errors (Coiera \& Alvarez, 2006). Over recent years, there have been significant advances in the development of technologies that support teamwork (Kuziemsky et al., 2009). VC, as a tool for improving communication between different levels of health care, has been described regarding a number of surgical subspecialties (Fleissig et al., 2006). Norum and Jordhoy published a study demonstrating the feasibility of VC for clinical and educational support between specialists at the University Hospital of North Norway and colleagues at the oncology and palliative care unit of the Nordland Hospital in Bodø, 300 miles apart. VC was a success for education and clinical case discussions with the remote oncologists in Bodø. During a 12-month period, 32 VCs were performed and this study demonstrated that telemedicine can be used for incorporating a remote palliative care unit into a university department (Norum \& Jordhoy, 2006). Dickson-Witmer et al. recently published a study of a VC network to discuss prospective patient management issues. Information was shared on a weekly basis regarding the discussion of treatment decisions and diagnostic procedures. $\mathrm{VC}$ led to an increase in National Cancer Institute treatment and the accrual of cancer control clinical trials (Dickson-Witmer et al., 2008). Three studies have been published on the experiences of breast cancer surgeons participating in IDTs. VC was compared to previous face-to-face clinical meetings through questionnaires, attendance, number of cases discussed, and anthropological analysis. Multidisciplinary case discussion can thus be facilitated by VC (Augestad \& Lindsetmo, 2009). Kunkler et al. proposed a comprehensive methodology to assess the clinical and economical effectiveness of VC in IDTs (Kunkler et al., 2005). This methodology was later tested in a randomized breast cancer trial (Kunkler et al., 2007), where 473 IDT patient discussions in two district general hospitals were cluster randomized to the intervention of telemedicine linkage to breast specialists in a cancer center, or to the control group of "in-person" meetings. VC was cost-effective, and breast cancer IDTs had clinical effectiveness similar to that of standard "in-person" meetings (Kunkler et al., 2007). There is a shortage of thoracic surgeons in the United Kingdom, and IDT meetings by VC were therefore introduced. The telemedicine meetings saved more than three working weeks of thoracic surgical time during the year (Davison et al., 2004). IDT meetings are used for establishing diagnoses; for tumor, node, and metastasis (TNM) classification; and for treating patients with head and neck tumors. In a Swedish study, telemedicine was introduced to link a regional hospital to two of the three district general hospitals. The conclusion was that costs could be saved by carrying out IDT meetings by means of telemedicine, instead of face-to-face meetings (Stalfors et al., 2005). A recent report on cancer services in Wales recommended an integrated cancer service using VC as a clinical tool. Regular IDT meetings reduced the need for patients to travel. They also increased access to expert opinion and reduced any delay in implementing treatment (Axford et al., 2002).

\subsection{Teleconsultation}

Expert consultation has been a key element of medical knowledge development and decision making for ages. In modern times, consultation is frequently needed for interpretation of diagnostic images. This is particularly important when dealing with rare diseases (e.g., congenital anomalies) or complex multidisciplinary conditions that require special management (Gackowski et al., 2010). Teleconsulting or telementoring has been accepted as a possible answer to upcoming higher quality demands by utilizing expert knowledge in everyday clinical routines (Seemann et al., 2010). In the case of complicated surgical procedures with limited training capabilities, teleconsulting has opened up new perspectives in surgery. Furthermore, intraoperative teleconsultation can support continuous training and medical education, which is of great importance in minimally invasive procedures, which require very specific knowledge and expertise (Seemann et al., 2010). Nowadays, many small cardiology units perform cardiac catheterization procedures or echocardiographic examinations far from referenced cardiosurgery centers. Experienced cardiologists can solve most hospitalized patients problems, locally. In particular cases, however, consultation with a cardiosurgeon is mandatory for optimal decision making (Gackowski et al., 2010). From a patient care perspective, medical patient consultations via video conferencing are now frequently used within the domains of dermatology, cardiology, wound-care, neurology, drug screening, diabetic training, and psychiatry (Krol, 1997). Video Consultation is considered useful for the following reasons (Ibrahim \& Fahim, 2009):

-e-doctors on health sites via video conference offer easy and almost immediate access.

-being unable to access an expert because of living in a rural area. It is often difficult for patients in rural areas to travel to large cities to seek medical advice in a tertiary hospital.

As a result, it is quite common for serious medical conditions to be diagnosed at a later stage.

-Patients may require further counselling when the treatment given to them at their first visit has failed.

The technology used to perform teleconsultation can range from a simple telephone, fax, or email, to satellite-based relay transfers, up to state-of-the-art computer and videoconferencing facilities (Angood, 2001).

In Brisbane, Australia, home-based speech treatment for 
Parkinson's disease is delivered remotely (Constantinescu et al., 2010). The telerehabilitaion system is able to capture high quality video $(640 \times 480$ pixel resolution) and audio, compressed at $384 \mathrm{kbit} / \mathrm{s}$ for later examination. A $128 \mathrm{kbit} / \mathrm{s}$ internet connection is being established between two videoconferencing systems using the public telecommunications network (ADSL). This connection enables videoconferencing at $320 \times 240$ pixel resolution between two systems. According to study performed by Constantinescu et al., patients achieve substantial improvements in vocal sound pressure levels during sustained vowel phonation, reading, and conversational monologues. Improvements are also perceived in the degree of breathiness and roughness in the voice, and in overall speech intelligibility during conversation. Patient are very satisfied with the audio and video quality of conferencing, and with online treatment overall. Telepsychiatry is becoming another specialty viewed as a reasonable alternative to office visits. Patients can be assessed, given psychological treatment, and prescribed medications from a distant site (Diamond \& Bloch, 2010). Hyler et al. (Hyler et al., 2005) carried out studies on a project included in their metanalysis, where 25 patients aged $4-16$ years were interviewed, once in-person, and once via telepsychiatry. They concluded that telepsychiatric interviewing produced the same quantity and quality of diagnostic information as I-P interviews. There are, however, concerns about using telepsychiatry in emergencies (Diamond \& Bloch, 2010). To date, studies on telepsychiatric assessment have relied exclusively on superiority-design comparisons of diagnostic reliability and comparability with in-person assessments, and have accepted the null hypothesis that there are no differences between assessment methods (Diamond \& Bloch, 2010). In Vienna, Austria, Seeman et al. have been working on a tele-endoscopy project using UMTS cellphones where arthroscopic video streams with lengths of $60 \mathrm{~s}$ for each sequence, showing endoscopic arthroscopies of the temoporomandibular joint or endoscopic assisted open reductions in mandibular head fractures, are hosted on a server and analyzed on a UMTS cellphone. Each of the arthroscopic video sequences are independently evaluated by two consultants and a medical expert in temporomandibular joint (TMJ) surgery. UMTS, the third generation and most recent telecommunication standard was developed for a more efficient use of existing frequency resources and, on the other hand, the need for a higher data transfer rate, and can thus be used to transmit videos (Horn et al., 1999). In this project all video streams are encoded in the H.263 standard with resolution of $176 \times 144$ pixels and a selected bandwidth of 56 $\mathrm{kbit} / \mathrm{s}$. The experiences of the 20 arthroscopic video streams showed that use of UMTS technology permits the transmission of video streams in the field of craniomaxillofacial surgery. Recognition of corresponding diagnoses, anatomy or arthroscopic situations had been observed at a high level. With the development of new technologies such as highspeed downlink packet access (HSDPA), higher data transfer rates than UMTS technology (currently $384 \mathrm{kbit} / \mathrm{s}$ ) become possible (see chapter 4.1 . for technical details). Also, the higher quality of mobile phone displays may contribute to image quality (i.e., higher colour depth and higher resolution) (Seemann et al., 2010). The digitization and transmission of real-time ultrasound images remains a major technical challenge. Bandwidths of about 70-100 Mbit/s are required to transmit ultrasound without compression. For this reason, teleultrasound has lagged behind other teleradiology applications. The vast majority of current teleultrasound systems rely on video compression, usually based on motion-compensated discrete cosine transforms (MC-DCT)(Chen et al., 1996).

Video compression predominantly causes a loss of high frequency data, distortion/displacement and alteration of pixel intensities/grey scale. There are currently no standards for teleultrasound image quality. The DICOM radiology image standard does not extend to video sequences apart from the storage of sequences as separate images, which is often impractical. There is, therefore little provision for the storage or transmission of ultrasound, uoroscopy, angiography or cine MRI. An effective standard for storage and transmission of ultrasound images is required for ultrasound to follow the rest of imaging into the digital age (Burgul et al., 2000). In Kuala Lumpur, Malaysia, a TeleUlstrasound was developed in response to the absence of a digital system, which enables the sharing of ultrasound images and data among radiologists, doctors, clinics, laboratories, and other medical officers who require this sort of data remotely, at any location in the country or the world. This system provides a Web interface with remotely accessed ".Net" Solution Imaging and Diagnostic, distributed application over the Internet for accessing and viewing information, while a Structured Query Language (SQL) database is deployed for data storage management. The ".Net" Solution technology enables a web-based distributed interface, which is server- and platform-independent across a network, without requiring preinstallation and tedious networking configuration. After log-in through a website this system determines the role specified by admin to the logged physician, and then directs the physician to his homepage, where he can view and examine the patient assigned to him and add his diagnosis. Patients can also log-in; however, this is limited to certain functions, such as viewing his diagnosis and updating his personal information (Hassan \& Ibrahim, 2010). In Krakow, Poland, Gackowski et al. developed Internet-based TeleDICOM software to make the teleconsultation of medical images possible. Interactive consultation between two or more centers offers real-time voice communication, visualization of synchronized Digital Imaging and Communications, and the use of interactive pointers and specific calculation tools. If direct interaction between physicians is not needed, the system can also be used in "offline" mode. This system is used for routine referral for cardiosurgical procedures (Gackowski et al., 2010). According to Gackowski et al. an optimal teleconsultation system should fulfill the following theoretical requirements: 
1) Sending and receiving of high-resolution DICOM images, from all available medical imaging modalities

2) Fast, real-time synchronized view of DICOM images and clips in 2 or more teleconsultation centers, avoiding delays during image loading

3) Live voice interaction of all participants

4) Availability of mouse-controlled pointers for real-tim e indication of a region of interest to other teleconsultation partners

5) Integrated fully automatic calibration of the diagnostic images, measurement tools and calculations for all available imagistic modalities

6) Secure and authorized access to medical data

7) Compatibility with standard computers and networks

8) Intuitive and easy to operate software

9) 24-h availability and stability of the system

10) Low cost

\subsection{Tele-education}

Telehealth applications are increasinlgy important for graduate and postgraduate education in the health professions, professional certification and recertification, contuniung medical eduacation, and health education for consumers and patients. Realizing telehealth's broad potential, for example, in telelearning, telemonitoring, telesurgical planning environments, telerobotic surgery, and teleconsultation, will allow forward-looking institutions to teach anything, anytime, anywhere with the same quality of curriculum and mentorship as delivered in traditional classroom settings, focusing on competence mastery rather than information mastery (Conde et al., 2010). In India, the majority $(70 \%)$ of medical experts live in the big cities. Thus, about $16 \%$ of the world's population, who reside in the rural areas of India, remain devoid of quality health care (Mahapatra et al., 2009). Therefore, telemedicine and teleeducation in health science, is gradually becoming adopted by the Indian Health System after decade-long pilot studies across the country. Sanjay Gandhi Postgraduate Institute of Medical Sciences (SGPGIMS), a tertiary-level academic medical center, has been piloting distance medical education projects using telemedicine technology. The SGPGI Telemedicine Program uses various high-bandwidth communication networks, including satellite-based communication, leased lines through a terrestrial fiberoptic network, and ISDN, to connect district hospitals in rural and remote areas, medical colleges, and tertiary-level hospitals for medical knowledge exchange. From Spetember 2001 to March 2002, SGPGIMS conducted a distance medical education program for postgraduate students of the SCB Medical College, through ISDN. In March 2003, permanent satellite communication links for point-to-point connectivity were provided at three medical colleges in Orissa, along with an advanced video-conferencing platform (Singh et al., 2004). Tele-education activities were carried out by four departments (Endocrine Surgery, Gastrointestinal Surgery, Gastronenterology, and Endocrinology) with AIMS, Kochi, which is on the southern coast of India located $2.500 \mathrm{~km}$ away. The Department of Urology at SGPGIMS started a virtual clinical grand round with their peers in two premier institutions of the country, located $800 \mathrm{~km}$ away, and through point-tomultipoint connectivity. SGPGIMS also established a connection with Ranguel University, Toulouse, France, the Holy Family Hospital, Rawalpindi, Pakistan and the Oregon Health and Science University, Portland, Oregon, through high-bandwidth ISDN. Between September 2001 and April 2009 , a total of 1.303 tele-educational sessions were carried out at SGPGIMS for the training and teaching of students, medical teachers, and practising doctors (Mishra et al., 2004). During the process of establishing an effective national teleeducational system, Conde et al. give eight essential recommendations:

1) Increase support for research in key areas including scalable, online, on-demand computional models for simulation that can be accessed from low-end computers; simplified software and hardware interfaces; software frameworks, artificial intelligence applications; remote 3-D visualization techniques.

2) Support for collaboratory centers to disseminate the use of telehealth technologies in training, education, and research.

3) Creation of a Palpable Human Project.

4) Establishment of national resource centers on virtual surgical trainers that focus on the development and testing of surgical simulations for training and education.

5) Facilitation of bandwidth access to underserved areas and institutions through high speed networks.

6) Implementation and provision of access to dynamic circuit networks technology.

7) Collaboration with professional societies in setting standard guidelines for the simulation of medical procedures.

\section{Telepresence Accelerating the Development of Telehealth Tools for Biomedical, Translational, and Clinical Research}

Telepresence in general means projecting virtual images of the operative field to remote sites (Satava, 1998). By using a telerobot to telecast their hand-motions to a remote operating room, surgeons perform operations without actually being with their patients (Ballantyne, 2002). Telerobotics was first developed with grants from the US Department of Defense to allow surgeons at remote locations to operate on wounded soldiers on the battlefield (Satava, 1995). Telepresence surgery offers a technological solution to surgical manpower shortages in remote and underserved areas. Moreover, it offers a means of improving outcomes for infrequently performed and technically-demanding operations. (Ballantyne, 2002). Examples of telepresence practice in telemedicine are: surgical telementoring, teledermatology, teleophtalmology, teletrauma, and emergency telemedicine. 


\subsection{Surgical Telemonitoring}

Telemonitoring is an active process and comprises the ability to guide, direct, and interact with another health care professional (in this case, a surgeon) in a different location during an operation or clinical episode. The level of interaction from the mentor can be as simple as verbal guidance while watching a transmitted real-time video of the operation (Challacombe, 2010). Surgery is, most of all, a visual specialty. Live pictures provide detailed information about anatomic landmarks, giving the mentor instant information about the patient's normal anatomy and pathological structures. Based on this instant information, the mentor can give advice to the operating surgeon and immediately correct his or her surgical actions (Augestad \& Lindestmo, 2009). Telementoring requires a secure highspeed connection with sufficient bandwidth to transmit a good picture and audio quality to the mentor's station. It has been shown that surgeons are generally able to compensate for delays of up to $700 \mathrm{~ms}$, but delays over $500 \mathrm{~ms}$ are quite noticeable (Fabrizio et al., 2000). If using an ISDN connection, a bandwidth of $384 \mathrm{kB}$ per second is needed to give sufficient picture quality for accurate interpretation by the mentor, although clinical work has been carried out using bandwidths as low as $128 \mathrm{~Kb}$ per second (Rosser et al., 1999). There is a knowledge gap between central and local hospitals, which is even more problematic in mainly rural countries, with community surgeons dispersed in remote corners of a large country (Anvari, 2007). The introduction of $\mathrm{VC}$ as an educational tool has led to a decrease in this knowledge gap. Until recently, the only proven technique for teaching surgeons new skills was one-site mentoring completed with hands-on course training and conferences. However, because of an overwhelming need for mentors/proctors and supporting evidence in the literature, telementoring is an application whose time has come (Augestad \& Lindsetmo, 2009). In order to illustrate the potential of telementoring in remote environments, a laparoscopic cholecystectomy was telementored from Yale University to a mobile surgery unit in Ecuador (Rosser et al., 1999), and in urology the Johns Hopkins team, in collaboration with an Italian group, successfully telementored remote surgeons in laparoscopic nephrectomy (Micali et al., 2000). More recently, a renal transplant surgeon who was a relative novice at laparoscopy was able to initiate independent hand-assisted laparoscopic donor nephrectomy by means of telementoring from an expert. Early results appeared to show that telementoring can significantly shorten the learning curve (Challacombe et al., 2005). The Johns Hopkins group successfully telementored a laparoscopic varicocelectomy and a percutaneous renal access for percutaneous nephrolithotomy between Baltimore and Sao Paulo, Brazil (Rodrigues et al., 2003). The remote surgeon controlled the laparoscope via an Automated Endoscopic System for Optimal Positioning (AESOP 3000, Intuitive Surgical, Inc., Sunnyvale, CA). This group has now carried out telesurgical telementoring in more than 17 cases using AESOP or the Percutaneous Access to the Kidney robot (PAKY, Johns Hopkins University, Baltimore, MD) (Bove et al., 2003). Besides the postgraduate tele-education mentioned in the Video-conferencing section, SGPGIMS also focuses on the training of medical professionals, and skill transfers by telemonitoring. The first successful experiment was carried out in 2004 when parathyroid surgery was done under expert guidance from SGPGIMS (Pradeep, 2006).

\subsection{Teledermatology}

The use of telemedicine has been described regarding many medical specialties, mainly for those in which imaging interpretation is a key step for diagnostic purposes. Dermatology is a specialty with a significant visual component, in a particularly favorable field for using of telemedicine. The application of teledermatology (as the use of telemedicine in dermatology is named) has been studied mainly as a form of distance medical care, modality known as teleconsultation. The use of teledermatology for distance consultation may be classified into two main groups. In the modality store-and-forward-system data referring to distance consultation, once sent, are stored in a database and are accessed after a variable time interval. In this modality, communication between the agents involved in the process occurs in a synchronic way. Digital photos of cutaneous lesions are usualy acquired under artificial light (fluorescent light). Images are then transferred from the camera to the computer using a USB cable, and stored in JPEG format (Joint Photographic Experts Group). On this issue, Seidenari et al. demonstrated that there was no significant difference in diagnostic accuracy between uncompressed images (tagged image file format, TIFF) and compressed ones (JPEG), showing that a factor 30 for compressed videomicroscopic images enables a good diagnostic accuracy (Seidernari et al., 2004). Files are then transmitted over telecommunication networks via e-mail or a specific web application. In 2003 a pilot study of the dermoscopicpathological approach using telediagnosis for melanocytic skin neoplasms revealed that the diagnostic accuracy reached $83 \%$ versus gold standard (conventional histopathological diagnosis by experts) (Ferrara et al., 2004). In a prospective analysis, where 60 patients were included in evaluating the role of teledermatology within a primary care system, the total agreement rate between live diagnosis and distant diagnosis was considered high, ranging from $86.6 \%$ to $91.6 \%$. When considering partial agreement, the figures went up to $98.3 \%$ $100 \%$ (Silva et al., 2009). Sharing images and comments on a given case together with other colleagues has been an incredible new tool and available for a few years on the net (www.telederm.org). This project is based on an Open Access Philosophy and a freely available teleconsultation service. On-line registration and password-protected log-in are needed. Once logged-in, users are immediately guided to the forum, which is characterized by high usability. Two moderators check the properties of the requests and contents. Dermatologists from all over the world can both see cases posted by other colleagues and express on-line their opinions 
on given cases or they can seek advice on their own patients. Cases are directly submitted to the discussion forum, and world class experts in that field answer the requests. To date, several hundred users have subscribed and over 2300 requests have been processed, each request received being on average, about 4 comments. Each day new cases are submitted and commented on (Massone et al., 2010). In some countries (i.e., New Zealand, Great Britain, the USA) a striking disparity between the dermatology workforce and the demand for melanoma screening has led to the commercialization of "teledennoscopy" by different companies. A patient arrives at a center to have pictures taken by a nurse or medical photographer who is trained by a dermatologist on how to capture the best images. This procedure obtains a detailed description of each lesion at both the clinical and dennoscopic levels. The nurse is trained to ask the patient a series of questions about his/ber skin and uses a low imaging threshold to avoid missing important lesions. Images and data are sent electronically to expert dennoscopists (teleconsultant) in a STF fashion. The dermoscopist's report, with provisional diagnosis and recommendations, is returned to the patient for selfmonitoring purposes or to the patient's primary care providers, or both, for referral for excision (Psaty \& Halpern, 2009). Modem PDAs and $3 \mathrm{G}$ mobile phones revolutionize the dimensions of data transmission, network coverage, and the number of pixels. As a result, technical limitations can be reduced to a minimum (Massone et al., 2008). A preliminary study demonstrates the feasibility and the potentiality of mobile teledermoscopy as a triage system for pigmented skin lesions (Meystre, 2005). Mobile teledermoscopy brings advantages for both physicians and patients. The former easy-to-use and lightweight tools that allow the rapid acquisition of suspected pigmented skin lesions images. These can be stored in a digital archive for follow-up control or dermoscopic-pathologic correlation, or sent to expert colleagues for a second opinion. This methodology only needs a new-generation cellular phone with a built-in camera, a demlatoscope suited for image acquisition, and a personal computer (Massone et al., 2010). In fact, one of the cardinal points of the e-Health program of the European Commission Information Society and Media is the prevention and management of diseases through research on "Personal Health Systems". The hallmark of this concept is to empower citizens to adopt an active role in managing their own health status and, in addition, to facilitate early diagnoses of diseases (Massone et al., 2007). In this context, mobile teledermatology and mobile teledermoscopy have the potential to become practical tools for everyone and may open the door for a new flexible triage system for the detection of skin cancer in general, and melanoma in particular. A high risk melanoma patient could be given a suitable modern cellular phone with an in-built digital camera and dermatoscope. In this way, suspected lesions could easily be followed-up using digital mobile teledermoscopy. Patients could be alerted via-SMS, reminding them of their molecheck e-visit (Massone et al., 2009).

\subsection{Teleophtalmology}

Teleophtalmology is an entity of telemedicine mostly used for screening retinopathy in diabetic patients. Throughout Canada, for example, diabetic retinopathy is common, with a prevalence up to $40 \%$ in people with diabetes (Ross et al., 2007). Up to a third of patients with diabetes do not receive an annual dilated eye examination by an ophthalmologist, despite universal access to health care (Tennant et al., 2007). In an effort to improve access, a teleophtalmology program was developed to overcome these barriers to eye care. In the teleophtalmology program, Alberta patients undergo stereoscopic digital retinal photographs following pupillary dilation. Digital images are then packaged into an encrypted password-protected compressed file for uploading onto a secure server. Images are digitally unpackaged for review as a stereoscopic digital slide show, and graded with a modified Early Treatment Diabetic Retinopathy Study (ETDRS) algorithm. Reports are then generated automatically as a PDF file and sent back to the referring physician ( $\mathrm{Ng}$ et al., 2009). Teleophthalmology programs in Alberta have assessed more than 5500 patients (9016 visits) to date. Nine hundred and thirty patients have been referred for additional testing or treatment. Approximately 2\% of teleophthalmology assessments have required referral for in-person examination due to ungradable image sets. Eye assessment by teleophthalmology, when compared to a screening examination, is according to $\mathrm{NG}$ et al. beneficial for three reasons ( $\mathrm{Ng}$ et al., 2009):

1) Unnecessary referrals are reduced or eliminated. As an example, diabetes patients with diabetic retinopathy need only be referred once they develop proliferative diabetic retinopathy (PDR) with high-risk characteristics or clinically significant macular edema (CSME).

2) Following treatment, patients can be followed up at a distance without the need for further travel. For example, having undergone focal laser treatment for CSME, a patient can be followed up by teleophthalmology and only requires an in-person clinical evaluation should they need re-treatment.

3) A comprehensive teleophthalmology examination enables the consultant to plan for necessary testing and treatment at the time of a patient's first visit. As an example, if a patient is found to have elevated intraocular pressure and enlarged cup-to-disc ratio, a visual field and diurnal tension curve can be ordered for the day of the patient's clinical referral. The teleophthalmology visit becomes the first contact visit with the patient, while the second "office" visit enables the ophthalmologist to review the findings of diagnostic testing and make expedient management decisions.

\subsection{Teletrauma and Emergency Care Telemedicine}

When applied to trauma, surgery and emergency care medicine, telepresence makes it possible for an experienced trauma surgeon or other specialist to assist or direct another 
less-experienced physician and/or surgeon who is operating or attending a patient at a distance. In order for true telepresence to be perceived by all participants, and thus have successful telepresence, it should have technology that creates an environment that mimics flawless motion, audio and video transmission (Latifi et al., 2007). In its nature, trauma requires fast, definitive and precise care, as well as major resources and continuous expertise. Trauma systems and major trauma centers have been shown to reduce mortality and morbidity (Trunkey, 2003), however, most trauma specialists and trauma centers around the world are concentrated within urban settings (Bran as et al., 2005). Subsequently, most of the population of the world is not covered by specialized trauma systems. Advanced technologies such as computers, diagnostic imaging, robotics, voice-activating machines, and remote controls have changed operating theatres in hospitals around the Western world (Latifi et al., 2007).

The first published example of true telesurgery was a transrectal ultrasound (TRUS)-guided prostate biopsy performed by Rosetta in Italy but due to the costs and complexity of the robotic procedure the benefits of telesurgery were outweighted (Rosetta \& Sala, 1995). The Arizona Telemedicine Program which uses broadband T1 line allows trauma surgeons to have video, audio and vital signs access to events unfolding in trauma and emergency rooms in remote emergency sites. This helps to guide physicians or nurses taking care of patients by being "virtually« present at the remote location (McNeill et al., 1998). In 2002, a collaboration between Johns Hopkins (Baltimore, MD) and Guy's Hospital (London, England) resulted in the first randomized controlled trial of telerobotic surgery (Challacombe et al., 2003) The group compared human with robotic and trans-Atlantic telerobotic percutaneous needle access using a validated kidney model into which a Kellet needle (Rocket Medical, Washington, England) was inserted 304 times. Half the insertions were performed by a robotic arm and the other half by urological surgeons. Order was decided randomly except for a subgroup of 30 Trans-Atlantic robotic procedures that were controlled by a team at Johns Hopkins via four ISDN lines. The robot was slower than the human but was more accurate both locally and remotely compared with human operators, as it needed less attempts for successful needle insertions (Challacombe et al., 2010). The possibility of true telesurgery arrived in the late 1990s with the introduction of the da Vinci and Zeus master-slave robotic systems. The surgical telerobot, which is positioned by the side of the patient, holds the camera and manipulates two or more surgical instruments. The surgeon and computer console can be positioned at the remote site. The surgeon acts as the «master» and the robot as the «slave» (Challacombe et al., 2010). In 2005, Colonel Noah Schenkman of the Walter Reed Army Medical Center performed live telesurgery (nephrectomy on two pigs) at the American Telemedicine Association, Washington, DC. (Hanley et al., 2005). This was the first telesurgery using the da Vinci surgical system, the first procedure to use stereoscopic surgical video streaming, and the first telesurgery over the Internet (Challacombe \& Wheatstone, 2010). Anvari et al. (Anvari et al., 2005) established a remote surgical service between Hamilton, Ontario, Canada, and North Bay General Hospital (North Bay, Ontario, Canada), some $400 \mathrm{~km}$ away. Using the Zeus system communicating through a redundant Internet protocol virtual private network (VPN) at a bandwidth of up to $15 \mathrm{MB}$ per second, the authors reported on 21 cases, including Nissen fundoplications and anterior resections. The transmission latency was $140 \mathrm{~ms}$, and the surgeon adapted to this easily. More recently, the da Vinci system has been modified and enabled for use over the Internet as well (Challacombe \& Wheatstone, 2010). The Level I trauma center of the City of Tucson, Arizona, the University Medical Center, in collaboration with Tucson Fire Department and Tucson Transportation Department launched Wireless Mobile Telemedicine and Telepresence in a prehospital setting by having video, audio, and data access from 17 Advance Life Support (ALS) ambulances of Tucson the Fire Department (TFD) since August of 2007. The City of Tucson Emergency Room Link or "ER-Link Tucson" project allows physicians to be virtually present at the scene and/or in the ambulance, while the patient is being transported to the trauma centre (Latifi et al., 2007). Through ER-Link, medical doctors at Tucson's University Medical Centre can use video and vital information telemetry to gain a sense of the severity of a patient's condition. This is achieved by viewing and in some cases speaking, to patients in real time from Tucson Fire Department ambulances en route to the hospital. All of the Department's 17 ambulances have been equipped with the ER-Link system. This system allows for constant two-way audio-video and near-constant medical data transmissions between ambulance personnel and the trauma and emergency room personnel. The communications are provided via regional traffic control and city communications infrastructure and wireless technology. Telepresence at the scene of an event is made possible from cameras mounted externally to the emergency vehicle. These cameras, in conjunction with the existing highway cameras (operating along the freeways or at intersections) provide command and control video to the regional 911 centres and emergency departments (Latifi et al., 2007). The clinical accuracy of telemedicine in evaluating trauma patients was assessed, when telemedicine was used for minor trauma consultation and compared with face-to-face consultations in two hundred patients. Skin-colour changes were accurately defined in $97 \%$, the presence of swelling or deformity in $98 \%$, diminished joint movement in $95 \%$, presence of tenderness in $97 \%$, weight bearing and gait $99 \%$, and radiological diagnosis was made correctly in $98 \%$ of cases (Tachakra et al., 2000). This application of telemedicine can make expert trauma care available to patients in hospitals and emergency rooms without advanced trauma systems, and potentially reduce costs, prevent unnecessary transfers, and promote early transfer when indicated. Telemedicine tools have also been applied to the field of wound care management where 
the sensitivity of remote diagnosis ranges from $78 \%$ for gangrene to $98 \%$ for identification of problem wound-healing respectively, whereas specificity ranges from $27 \%$ for erythema to $100 \%$ for ischemia (Wirthlin et al., 1998). Telemedicine will become a major tool in trauma care and trauma education. Trauma resuscitation can be performed successfully and safely using telemedicine principles, when guided by and under direct supervision of a trauma surgeon (Latifi et al., 2007).

\section{The Technology Aspects of Telemedicine}

Using up-to-date technology is of crucial importance for telemedicine. This can be divided into two main elements (Austerberry, 2005):

1) Peripherals, digital video cameras, microphones, speakers, monitors or even special equipment such as digital microscopes with built-in digital cameras, video endoscopes, ultrasound imaging devices.

2) Transmission of voice and video signals, where transmission is regarded as a process of sending, propagating and receiving an analogue or digital information signal over a physical point-to-point or point-to-multipoint transmission medium, either wired, optical fibre or wireless. The core technology used in transmission systems, especially audio and video signals, is the digital compression of audio and video streams in real-time.

\subsection{Peripherals}

In order to transfer images over a distance, a digital video or still camera is needed for capturing, and for visualisation on a screen. There are several different industrial standards for such devices depending on the purpose and image quality required by such a device. The quality of the image the camera is able to capture and transfer to another device, such as the monitor, depends on the components from which it is built. The digital camera generally consists of three elements: lens, image sensor, and electronics for the image processing, encoding, and transmission of the image. It is important that the individual elements are properly aligned. The industry is able to produce high-quality cameras of extremely small sizes. They are used for example, in installed endoscopic devices. Cheap or simple camera models can be used in simple application devices for teleconferencing. It is important that the camera has the option to focus on an image automatically in the case of moving objects or entities in space. Autofocus systems rely on one or more sensors to determine correct focus. Simple camera models have a builtin single sensor to capture images. The brightness of the image is automatically adjusted by the electronic circuit. Such cameras are often used for remotely controlling a patient during surgery, for teleconsulting (as mentioned in 2.2.), and to transfer images from the operating room to the classroom for students. Camera image quality depends on the size and resolution of the image sensor (a device that converts an optical image to an electrical signal). The image sensor consists of millions of special type transistors sensitive into light integrated on one chip. Today, the industry uses two types of image sensors for cameras: CCD (charge coupled device) or CMOS (complementary oxide semiconductor). Both technologies have advantages and disadvantages regarding image quality. Image quality is usually delivered by a resolution image, which is expressed in pixels. Examples of standard image sizes are: 320x240, 640x480, 720x576, 720x480, 1440x720, and 1920x1080. Some high-end still-camera models can capture at a resolution of $8984 \times 6732$ pixels. The quality of the integrated lens is of great importance. They are divided into fixed focal length lenses and zoom lenses, where the focal length of the lens is changeable. In general, fixed focal length lenses overall give better image quality and they allow for capturing images under critical low-light conditions. Additional lighting is provided under low-light conditions. A 3-CCD camera is used instead of single chip CCD for better picture quality. 3-CCD imaging system uses three separate chargecoupled devices, each one taking separate captures of red, green, or light blue. Light coming into the lens is split by a prism, into the separate wavelength lights (R-red, G-green, B-blue) which are then directed in CCD sensors. The captured image is next processed in the electronic camera module and occupies a certain memory space. A video clip includes a sequence of successive $25 \mathrm{p}$ (progressive) or $50 \mathrm{i}$ (interlaced) captured images, the quantities of data being measured in Mbs. The volume of data transfer (and storage space) affects the number of pixels in the individual image frame, and the number of captured images in each second. Normally, the non-compressed data amount from a CCD sensor is too high for transmission. Compression reduces the number of bits used to represent each pixel in the image. Codec's (compression/ decompression algorithm) is used for the compression process. Some of the more frequently used video codec's today are DV, MPEG1, MPEG2, MPEG4, DIVIX, and others. The goal of compression is clear: how to reduce the data amount as much as possible in order to keep the image/sound quality as high as possible. Telephone lines are one of the media through which we can transmit video signals, but with some limitations. Data transfer is limited by the so-called bandwidth. The transfer of video via telephone links initially allowed a theoretical bandwidth rate of 56 $\mathrm{kbit} / \mathrm{s}$, and $128 \mathrm{kbit} / \mathrm{s}$ later for ISDN. Video transmission was limited to low-resolution image. The standard defines the screen sizes of the Common Intermediate Format (CIF) $352 \times 288$, and Quarter CIF (QCIF) to be of 176x144 pixels. The number of progressively-scanned frames transmitted each second was limited to 8 fps. After applying sophisticated DSP technology and by using a wider bandwidth, it was possible to achieve transmission data rated from 1.5 to $8 \mathrm{Mbit} / \mathrm{s}$. Today technology allows for the transferring of high-quality video and audio data over extremely short delay times (latency). If there is no guaranteed fixed bandwidth, the latency can be extended. 
The problem of packet loss arises if there is no guaranteed fixed bandwidth. The video and audio become disjointed. This problem is solved by adaptive streaming technology. In the case of network saturation, the ratio of video compression increases and vice versa. Once the network is re-released, the volume of data transferred can be increased again. The alternative to a copper or fibre connection is a microwave connection. This could be a satellite. Very small aperture satellite antenna offer one or two-way connection links to the internet. In Europe this service is offered by the Astra satellites. This service includes point-to-point (fixed or portable stations), point-to-multipoint (star configuration), and meshed networks. The next alternative to fixed lines is to use wireless communication, through cellular networks covering large territories. Mobile networks have a long way to go before achieving a fixed wired transmission capacity, but they have two advantages mobility, and availability. The second-generation digital phones were limited to about 14 $\mathrm{kbit} / \mathrm{s}$ data rate. The third-generation $(3 \mathrm{G})$ offers a theoretical data rate up to $2.4 \mathrm{Mbit} / \mathrm{s}$. In both Europe and the wider world these is great anticipation about the introduction nextgeneration networks (NGN), including the important place occupied by the next-generation of mobile networks (NGMN). In Europe there is already intense transition from analogue to digital TV. The switchover from analogue to digital terrestrial TV will free-up an unprecedented amount of spectrum in the $800 \mathrm{MHz}$ field - the Digital Dividend. Allocating some of the Digital Dividend spectrum to mobile broadband will allow mobile operators to provide broadband services to everyone, even in rural areas. International Mobile Telecommunications (IMT) includes a set of standards for a variety of multimedia mobile networks (EDGE, UMTS, DECT, WiMAX). Different standards (HSDPA, HSUPA, HSP+, LTE) allowing for transfer speeds up to $50 \mathrm{Mbs}$. For the fourth-generation network, ITU requires download transmission speeds of up to $1 \mathrm{Gbit} / \mathrm{s}$. How will all this technology help telemedicine? Not long ago, mobile phones only had small screens with extremely low resolution. Today's cell phones already have a much larger screen with high-resolution, and development trends are moving in this direction continuously. Many of these mobile devices - also known as smart mobile devices (smart phones) also have touch screens and the ability to increase a certain part of the picture. Smart mobile phones with touch screen web browsers have a built-in big, high resolution screen, giving access to the Internet. This phone can also be used as a camcorder, because of the built-in camera. The qualities of built-in mobile phone cameras are constantly being improved, but they still lag behind traditional digital cameras.

\subsubsection{Digital Video Camera}

A digital camera (still or video) is a camera that digitally takes either video or still images or both, by capturing images via an electronic image sensor. Commercial digital cameras recording of a sound is standard. Digital cameras are incorporated into many devices ranging from PDAs to mobile phones. A digital camera can be divided into two segments, compact cameras and DSLR cameras. Both of them can be used for still image and/or video capturing. Both types of camera can be used for documentation and be directly connected via cable for the immediate transfer of stills or video to distance locations. Images (stills) can be stored using lossy compression JPEG format. Many DSLR cameras, especially professional, support a RAW image format which is an unprocessed set of pixel data directly from the camera's sensor. The formats for movies are AVI, MPEG, MOV, and WMV. The video resolution extends from $640 \times 480$ pixels to HD $1920 \times 1080$ pixels, with a frame rate from 15 to $50 \mathrm{fps}$. There are some commercially-available models with capturing speeds up to 1000 fps. Many cameras store the pictures into memory cards. The more-widely used are SD memory cards having storage capacity from $128 \mathrm{MB}$, and up to 32 GB. Some DSLRs use SD and Compact Flash (CF) with storage capacities up to 128 GB of memory. Digital still or video cameras use either a CCD image sensor or a CMOS sensor. The task of a sensor is the capturing of light and converting it into electrical signals. It is difficult to give advantage to either CCD or CMOS types of sensor. Some professional DSLR cameras with built-in CMOS image sensors are also widely used in professional video production. Cameras can capture video in HD resolution of $1920 \times 1080$ pixels by $25 \mathrm{fps}$, but they suffer against the rolling-shutter effect. Professional video cameras are focused in order to provide high-quality video images. They can have either one single sensor or a triple sensor. Most of triplesensor designed cameras utilize an optical prism block directly behind the lens. This prism block divides incoming light into the three primary colours, red $(R)$, green $(G)$, and blue (B), directing each colour into a separate $\mathrm{CCD}$ or CMOS image sensor mounted on each face of the prism (Austerberry, 2005). The cameras are able to produce a higher-resolution image, with better colour fidelity than is normally possible with just a single video pickup. In both single-sensor and triple-sensor designs, the weak signal created by the sensors is amplified before being encoded into analogue signals for use by the viewfinder and monitor outputs, and also encoded into digital signals for transmission and recording. The analogue outputs are normally in the form of either a composite video signal, which combines the colour and luminance information into a single output; or an R-Y, B-Y, Y component video output through three separate connectors. Some special camera models for industry or medical use are very compact but also use 3-chip Full HDTV Camera head with various available outputs: $720 \mathrm{p}, 1080 \mathrm{i}$ and 1080p. The last sign i-interlace or p-progressive denotes either interlaced (the odd then even lines of image frame are scanned alternately) and progressive, where an image frame is scanned top-to-bottom over a pass. This interlaced manner is used for the reducing the amount of data. It was first designed for display on CRT televisions. Each picture is separated into two fields: the "top field," which has the odd numbered horizontal lines, and the "bottom field," which has the even-numbered lines. After reception/decoding, the two 
fields are displayed alternately with the lines of one field interlacing between the lines of the previous field. This format is called interlaced video; two successive fields are called a frame. The typical field rate is then 50 (Europe/PAL) or 59.94 (US/NTSC) fields per second (Austerberry, 2005). If the video is not interlaced, then it is called progressive video and each picture is a frame.

\subsubsection{Displays}

High-definition video displays are needed for medical applications when delivering accurate colour reproduction (dermatology). The monitor should display natural images with minimal ghosting. The screen format ranging from 15 ins up to 40 ins. It should deliver a 1929x1200 pixels HDTV quality with full colour $(16.7$ million $)$ graduated reproduction. The brightness performance must be as high as possible. The ingoing signals to the monitor can be analogue or digital. It is useful to have robust interface connectors. Today's standard for input signals are composite signals, RGB signals, HDTV 1080 HD signal, and digitals HD-SDI or SD-SDI signals.

\subsection{Transmission of Voice and Video Signals (Video compression)}

Audio, video, and image signals require a vast amount of data for their representation. There are many reasons why the data must be compressed, such as large storage requirements, slow storage (playback in real time), and because of the network bandwidth (bottleneck). Compression reduces the number of bits used to represent each pixel in the image. The compression system exploits the mechanisms of human visual perception to remove redundant information, but still produces a compelling viewing experience. A video is a sequence of picture frames, usually 24 fps (film industry), 25 pfs (PAL video system) or $30 \mathrm{fps}$ (NTSC video system). The most used compressing format for stills is JPEG, it is a lossy format based on technique called the discrete cosine transform (DCT) (Furht et al., 1995). A restoration procedure does not exist to present an original picture back from a JPEG. The standard supports different levels of compression. The compression of a single frame in video is called intraframe - compression within the frame. In a sequence of video images there is a little change from one picture to the next. Transmitting only the differences between successive pictures can produce a large reduction in video data. This procedure is called temporal or inerframe compression. It allows for 3:1 reduction over a interframe compression. Compression can be lossless or lossy. In lossless compression all original data can be retrieved. In video compression, where a higher level of compression is used, the lossless codec's would be insufficient. Most video compression is lossy. Video compression is a trade-off between disk space, video quality, and the cost of hardware required to decompress the video in a reasonable time. The human visual system has psycho-visual redundancy - not all visual information is treated with the same relevance. An example is lower accuracy to colour detail than luminance. A common practice for reducing the data rate is to "thin out" or subsample the two chrominance planes. In effect, the remaining chrominance values represent the nearby values that are deleted. This works because the eye better resolves brightness details than chrominance details. The 4:2:2 chrominance format indicates that half the chrominance values have been deleted, while the 4:2:0 chrominance format indicates that three quarters of the chrominance values have been deleted (Austerberry, 2005):. If no chrominance values have been deleted, the chrominance format is $4: 4: 4$. The compressing codecs must be used for compressing the video. These codecs belong either to the international standards, open standards or proprietary standards. Some the most frequently used codecs are (Austerberry, 2005):

1) H.261 - videoconferencing codecs formulated under the ITU for videophones and videoconferencing over ISDN lines. One of the demands of H.261 is the real-time operation. The standard defines screen size of CIF $352 x$ 288 and QCIF of $176 \times 144$ pixels. It uses progressive scan and 4:2:0 sampling. Data rates from $64 \mathrm{kbit} / \mathrm{s}$ up to $2 \mathrm{Mbit} /$ as are supported by the standard.

2) $M P E G-1$, is the first standard by the multimedia community and is a standard for lossy compression of video and audio. This standard has long been used for audio and video presentations on CD-ROMs. It is designed for storage based applications at data rates of $1.5 \mathrm{Mbit} / \mathrm{s}$. It does not support streaming.

3) H.263 -is developed from H.261 for low-bit rate applications. It solves the problem of operating at 28 $\mathrm{kbit} / \mathrm{s}$ and can be used for videophone applications.

$M P E G-2$ is a standard aimed at higher resolution (up to 40 $\mathrm{Mbits} / \mathrm{sec}$ ), a high-quality system for broadcasting television and intended to replace analog composite systems for digital transmission systems. It is also used for DVD recording systems.

$M P E G-4$ is a patented collection of methods defining the compression of audio and visual digital data. It is the first MPEG system to support streaming. The format is designed to support a wide range of bit dates, from $5 \mathrm{Kbit} / \mathrm{s}$ up to 50 Mbit/s. This allows it to low-bit rate wireless data through to HDTV applications.

\subsubsection{Streaming the Video over Internet}

The first task of the internet was delivering only data. The first audio and video applications used the Internet only as media for file transfer. A computer needed to download the complete file to the disk before it could play them. As soon as the slow analog telephone connections were replaced with xDSL, and with higher data transfer speeds, the delivery of multimedia files (especially video) in real-time over IP became a reality (Skalar, 2000). Streaming technology now allows playing of audio and video files immediately after being transmitted on the internet, in real-time. The User Database Protocol (UDP) instead of Transmission Control Protocol (TCP) is used for streaming technologies. The difference between the two protocols is how they check for errors. Streaming needs a transmission protocol that can ignore data errors. One of the first applications used the real-time was video-conferencing. Video conferencing codecs started with H.261 followed by H.263 
codecs and the latest advanced video codecs

H.264. The time latency (propagation delay of audio and video signals) is an important factor in video-conferencing, especially in telemedicine. A webcasting system uses the same real-time protocols, where the latency of some seconds doesn't play a role. Another way to distribute audio and video files over IP is on-demand. The audio and video content is streamed on-demand whenever a client seeks for the content. This service is very popular in advertising, product and sales training, entertainment, Tele-education and medicine (as already mentioned in 2.3). The streaming procedure can be realized in four steps:

1) Microphone

2) Video camera: audio and video capture and encoding (compression)

3) Server: storage and streaming

4) Network: distribution

Computer on site: Media player plays the content Video cameras and microphones are used for capturing the audio/video. Most commercial models of video cameras already have built-in microphones. The microphone or surrounding noise level is too high for special occasions where the sound quality is of higher importance. In that case we need to set the microphones close to the speakers as much as possible; this eliminates the reception of disturbing surrounding noise. If there are more speakers in a larger room, we need one microphone, a lavaliere type, for each speaker. Lavalieres are small and lightweight microphones which do not disturb the speaker. The microphones are connected by wire, or are wireless, where the digital audio signal is radio transferred to the mixer station. Many cameras are usually installed in typical surgical theatres. The outgoing signals from the camera (video/audio) can be analog or digital. It is preferable to use robust interfaces that use coaxial cable and connectors. Analog composite uses 75 Ohms coaxial with BNC connectors, but industry uses RCA connectors for consumer cameras. Many low-cost video monitors are only equipped with composite inputs, but professional monitors provide BNC connectors. The next analog video signal is SVideo or $\mathrm{Y} / \mathrm{C}$, which uses a small 4-pin connector. The IEEE 1394 (FireWire) is a IEEE Standard, and now widely used by many manufacturers to transfer digital video/audio (and remote control signals) data up to $800 \mathrm{Mbit} / \mathrm{s}$. There are two versions of these connectors, a six-pin and a four-pin. HDMI (HighDefinition Multimedia Interface) is a compact audio/video interface for transmitting non-compressed digital data. HDMI connects digital audio/video sources such as video cameras, digital still cameras, computer monitors, and graphic computer interfaces. A video capture card installed in the computer converts analog or digital video and audio into the AVI format. Next, the video/audio file in AVI format is compressed with codec. Different codecs use several methods to compress the video. The result of this compression is smaller amounts of video/audio data, which reduces the need for the network bandwidth. The original audio/video data rate of a DV camcorder (where video in compressed 5:1) is $25 \mathrm{Mbit} / \mathrm{s}$, and the data rate for studio video cameras can reach $350 \mathrm{Mbit} / \mathrm{s}$.

\subsection{D Imaging Technologies in Medicine}

Three-dimensional (3D) visualisation adds a new dimension to presenting video content. 3D has the ability to visualize human internal organs in their true form and shape (White, 2006). 3D Imaging technologies not only make is possible to visualize, but also to analyze and manipulate $3 \mathrm{D}$ structures from the captured 3D image. This is significant for a number of diagnostic and therapeutic applications. The importance of 3D technology is obvious. At the end of 2009, at the Radiological Society of North America's Scientific Assembly and Annual Meeting (NVIDIA and Siemens Healthcare) a new, 3D ultrasound viewing experience was demonstrated that enables expectant parents and their medical caregivers to view the fetus in incredible detail using 3D Vision-ready LCD, and NVIDIA 3D Vision glasses to demonstrate how patients and their doctors can view remarkable, high-resolution, three-dimensional sonograms in true 3D. Results of recent developments in three-dimensional screens give the possibility to presenting $3 \mathrm{D}$ video content, where special $3 \mathrm{~d}$ glasses are no longer required. Efficient 3D content creation and media technology, such as VoD (Video on Demand), could all be in place within the next few years. We need new technologies for capturing or presenting images in 3D. Although stereoscopic vision was first presented in the 18th century, digital technologies have brought new extensions. In traditional stereo vision, two cameras, displaced horizontally from one another were used to obtain differing views of a scene, in a manner similar to human binocular vision. Although most image capturing cameras are based on a twin-lens system, the latest achievements of industry show a new professional camera which allows the recording of $3 \mathrm{D}$ images using just a single lens. We need double memory and special $3 \mathrm{D}$ camera/3D vision screens or glasses for 3D image visualisation. Glasses have always been seen as a major drawback to $3 \mathrm{D}$ on televisions. In order to visualise $3 \mathrm{D}$ images on $2 \mathrm{D}$ monitors, there are different technologies, such as anaglyph technology (anaglyph images are used to provide a stereoscopic 3D effect), polarisation technology, or auto stereoscopy technology.

\section{References}

[1] Angood, PB. (2001). Telemedicine, the Internet, and world wide web: overview, current status, and relevance to surgeons. World J Surg, 25, 11, (Nov 2001) 1449-1457.

[2] Austerberry, D., (2005). The Technology of Video \& Audio Streaming, Focal Press, ISBN-13: 978- 0240805801, Burlinghton.

[3] Anvari, M., McKinley, C., Stein, H. (2005). Establishment of the world's first telerobotic remote surgical service for provision of advanced laparoscopic surgery in a rural community. Ann Surg, 241, 3, (Mar 2005), 241:460-464.

[4] Anvari, M. (2007). Telesurgery: remote knowledge translation in clinical surgery. World J Surg, 31, 8, (Aug 2007) 1545-1550. 
[5] Augestad, KM., Lindsetmo, RO. (2009). Overcoming distance: video-conferencing as a clinical and educational tool among surgeons. World J Surg, 33, 7, (Jul 2009) 1356-65.

[6] Axford, AT., Askill, C., Jones, AJ. (2002). Virtual multidisciplinary teams for cancer care. $J$ Telemed Telecare, 8 , Suppl 2, (2002) 3-4.

[7] Ballantyne, GH. (2002). Robotic surgery, telerobotic surgery, telepresence, and telementoring. Review of early clinical results. Surg Endosc, 16, 10, (Oct 2002) 1389-402.

[8] Bove, P., Stoianovici, D., Micali, S., et al. (2003). Is telesurgery a new reality? Our experience with laparoscopic and percutaneous procedures. $J$ Endourol. 17, 3, (Apr 2003) 137-142.

[9] Branas, CC., MacKenzie, EJ., Williams, JC., et al (2005). Access to trauma centers in the United States. JAMA, 293, 1, (Jun 2005) 2626-2633.

[10] Burgul, R., Gilbert, FJ., Undrill, PE. (2000). Methods of measurement of image quality in teleultrasound. Br J Radiol, 2000 Dec; 73(876):1306-12.

[11] Challacombe, BJ., Kavoussi, LR., Dasgupta, R. (2003). Transoceanic telerobotic surgery. BJU Int, 92, 7, (Nov 2003) 678680 .

[12] Challacombe, B., Kandaswamy, R., Dasgupta, P., Mamode, N. (2005). Telementoring facilitates independent hand-assisted laparoscopic living donor nephrectomy. Transplant Proc, 37, 2, (Mar 2005) 613-616.

[13] Challacombe, B., Patriciu, A., Glass, J., et al. (2005). A randomized controlled trial of human versus robotic and telerobotic access to the kidney as the first step in percutaneous nephrolithotomy. Comput Aided Surg, 10, 3, (May 2005) 165-171.

[14] Challacombe, B., Wheatstone, S. (2010). Telemonitoring and Telerobotics in Urological Surgery. Curr Urol Rep, 11, 1, (Feb 2010) 22-28.

[15] Chen, W., Gupta, S., Turner, J. (1996). Motion-compensated discrete-cosine transform as the enabling technology for video conferencing and telemedicine. Telemed J, 2, 4, (Winter 1996) 313-7.

[16] Chen, W., Turner, J., Crawford, C. (1996). The process of elimination: video compression in telemedicine. Telemed, $J 2$, 1, (Spring 1996) 36-41.

[17] Coiera, E., Alvarez, G. (2006). Interdisciplinary communication: An uncharted source of medical error? Journal of Critical Care, 21, 3, (Sep 2006) 236-242.

[18] Conde, JG., De, S., Hall, RW., Johansen, E., Meglan, D., Peng, GC. (2010). Telehealth innovations in health education and training. Telemed $J E$ Health. 16, 1, Jan 2010) 103-6.

[19] Constantinescu, GA., Theodoros, DG., Russell, TG., Ward, EC., Wilson, SJ., Wootton, R. (2010). Home-based speech treatment for Parkinson's disease delivered remotely: a case report. J Telemed Telecare. 16, 2, (Dec 2010) 100-4.

[20] Davison, AG., Eraut, CD., Haque, AS. et al. (2004). Telemedicine for multidisciplinary lung cancer meetings. $J$ Telemed Telecare, 10, 3, (2004) 140-143.
[21] Diamond, JM., Bloch, RM. (2010). Telepsychiatry Assessments of Child or Adolescent Behavior Disorders: A Review of Evidence and Issues. Telemed $J$ E Health, 16, 6, (Jun 2010) 1-5.

[22] Dickson-Witmer, D., Petrelli, NJ., Witmer, DR. et al. (2008). A statewide community cancer center videoconferencing program. Ann Surg Oncol, 15, 11, (Nov 2008) 3058-3064.

[23] Fabrizio, MD., Lee, BR., Chan, DY., et al. (2000). Effect of time delay on surgical performance during telesurgical manipulation. J Endourol, 14, 2, (Mar 2000) 133-138.

[24] Ferrara, G., Argenziano, G., Cerroni, L. (2004). Cusano F, Di Blasi A, Ursa C el til. A pilot stUdy of a combined dermoscopic-pathological approach to the telediagnosis of melanocytic skin neoplasms. J Telemed Telecare, 10, 1, (2004) 34-8.

[25] Fleissig, A., Jenkins, V., Catt, S., Followfield, L. (2006). Multidisciplinary teams in cancer care: are they effective in the UK? Lancet Oncol, 7, 11, (Nov 2006) 935-943.

[26] Furht, B., Smoliar S., W., HongJiang Z., (1995). Video Image Processing in Multimedia Systems, Kluwer Academic Publishersr, ISBN-13: 978-0792396048, Norwell.

[27] Gackowski, A., Czekierda, L., Chrustowicz, A., Cała, J., Nowak, M., Sadowski, J., Podolec, P., Pasowicz, M., Zieliński, K. (2010). Development, Implementation, and Multicenter Clinical Validation of the TeleDICOM-Advanced, Interactive Teleconsultation System. J Digit Imaging, (May 2010).

[28] Hanley, EJ., Miller, BE., Herman, BC., et al. (2005). Stereoscopic robotic surgical telementoring: feasibility and future applications. 10th Annual Meeting and Exposition of the American Telemedicine Association, Denver, CO, April 2005. Telemed and E Health, 11, 2, (Apr 2005) 247.

[29] Hassan, A., Ibrahim, F. (2010). Development of a Kidney TeleUltrasound Consultation System. J Digit Imaging. (Apr 2010).

[30] Heinmann, GD. \& Zeiss, AM. (2002). Team performance in health care: Assessment and development. Heinmann, GD. \& Zeiss, AM. (Ed.), Springer, ISBN: 978-0306467073, Kluwer.

[31] Horn, U., Stuhlmuller, K., Link, M., Girod, B. (1999). Robust Internet video transmission based on scalable coding and unequal error protection. Signal Process, 15, (Jul 1999) 77-94.

[32] Hyler, SE., Gangure, DP., Batchelder, ST. (2005). Can telepsychiatry replace in-person osychiatric assessmnets? A review and meta-analysis of comparison studies. CNS Spectrums, 10, 5, (May 2005) 403-413.

[33] Ibrahim, K., Fahim, S. (2009). Cooperative Remote Video Consultation on Demand for e- Patients. J Med Syst, 33, 6, (Dec 2009) 475-483.

[34] Kitamura, C., Zurawel-Balaura, L., Wong, RKS. (2010). How effective is video consultation in clinical oncology? A systematic review. Curr Oncol, 17, 3, (June 2010) 17-27.

[35] Krol, M. (1997). Telemedicine. IEEE Potentials, 16(4), 29-31, October/November. 1997. 
[36] Kunkler, IH., Fielding, RG., Brebner, J. et al. (2005). A comprehensive approach for evaluating telemedicinedelivered multidisciplinary breast cancer meetings in southern Scotland. J Telemed Telecare. 11(Suppl 1), (2005) 71-73.

[37] Kunkler, IH., Prescott, RJ., Lee, RJ. et al. (2007). TELEMAM: a cluster randomised trial to assess the use of telemedicine in multi-disciplinary breast cancer decision making. Eur J Cancer, 43, 17, (Nov 2007) 2506-2514.

[38] Kuziemsky, CE., Borycki, EM., Purkis, ME., Black, F., Boyle, M., Cloutier-Fisher, D., Fox, LA., MacKenzie, P., Syme, A., Tschanz, C., Wainwright, W., Wong, H. (2009).
Interprofessional Practices Team. An interdisciplinary team communication framework and its application to healthcare 'eteams' systems design. BMC Med Inform Decis Mak, 15, 9, (Sep 2009) 43.

[39] Latifi, R., Weinstein, RS., Porter, JM., Ziemba, M., Judkins, D., Ridings, D., Nassi, R., Valenzuela, T., Holcomb, M., Leyva, F. (2007). Telemedicine and telepresence for trauma and emergency care management. Scand J Surg, 96, 4, (2007) 281-9. 